\title{
Drenagem PROFILÁTICA DE ROTINA DE ANASTOMOSES EM CIRURGIA COLORRETAL ELETIVA. REVISÃO SISTEMÁTICA DA LITERATURA E METANÁLISE
}

\author{
Eliane Camargo de Jesus, Delcio Matos*, Aldemar de Araújo Castro
}

Trabalho desenvolvido na Disciplina de Gastroenterologia Cirúrgica do Departamento de Cirurgia e na Unidade de Ensaios Clínicos e Metanálise do Centro Cochrane do Brasil, UNIFESP. Escola Paulista de Medicina, S. Paulo, SP.

RESUMO - Objetivo. Analisar os resultados da comparação entre 0 uso ou não de drenagem profilática de rotina de anastomoses em cirurgia colorretal eletiva, testando a seguinte hipótese: 0 uso de drenagem profilática rotineira de anastomoses em cirurgia colorretal eletiva não é justificável.

UnITERMOS: Drenagem cirúrgica. Cirurgia do cólon e reto. Revisão acadêmica. Metanálise. Anastomose cirúrgica.

\section{INTRODUÇĀO}

Robert Lawson Tait, citado por Berliner, 1964, escreveu uma frase que tem sido repetida por vários cirurgiões até os dias atuais: "quando em dúvida: drenar", que reflete a falta de evidência científica para a tomada de decisão quanto da utilização ou não de drenos profiláticos em anastomoses na cirurgia colorretal.

Tendo em vista que na prática a colocação ou não de dreno implica custos adicionais, cuidados pós-operatórios especiais e grande desconforto ao paciente, a elucidação de tal questão torna-se de grande relevância.

Estudos experimentais ${ }^{1-4}$ sugerem que a drenagem de uma anastomose, quando não se tem dúvidas quanto a sua integridade, tensão ou irrigação sangüínea, pode efetivamente colocar em risco a anastomose. Ensaios clínicos randomizados ${ }^{5-12}$ não mostraram diferenças nas taxas de complicações pós-operatórias ao realizar a comparação da drenagem profilática rotineira de anastomoses após cirurgia colorretal eletiva com a não utilização de drenos.

Diante do fato de haver vários ensaios clínicos randomizados, comparando drenagem profilática das anastomoses colorretais com a não drenagem, porém incapazes de validar esse procedimento, poder-se-ia sugerir a realização

\footnotetext{
* Correspondência:

Rua Napoleão de Barros, 610 - Vila Clementino São Paulo - Brasil - CEP: 04024-002

Tel/Fax: (11) 50847551

E-mail: dmatos.dcir@epm.br
}

de um ensaio clínico bem planejado,com amostra de tamanho adequado, com poder de detectar possíveis diferenças entre as intervenções a serem testadas, proporcionando assim resultados confiáveis ${ }^{13,14}$.

Esse tipo de estudo, entretanto, poderia apresentar várias dificuldades técnicas e ou financeiras, necesssitar de um longo tempo de seguimento, o que nos fez optar pela realização de uma revisão sistemática da literatura e metanálise, modelo de investigação que é aceito, atualmente, como a melhor forma de se obter evidência científica de melhor qualidade para se avaliar decisões relacionadas com a conduta terapêutica ${ }^{15}$. Assim, o nosso objetivo foi analisar os resultados da comparação entre o uso ou não de drenagem profilática de rotina de anastomoses em cirurgia colorretal eletiva. A hipótese testada foi que a drenagem profilática rotineira de anastomoses em cirurgia colorretal eletiva não é justificável.

\section{Métodos}

O método de pesquisa utilizado foi uma revisão sistemática com metanálise dos ensaios clínicos publicados ou não na literatura, após elaboração de um protocolo de revisão sistemática conforme orientação do Cochrane Collaboration Handbook ${ }^{16}$, e aprovação pelo corpo editorial do Grupo de Câncer Colorretal da Cochrane, Bispebjerg Hospital, Copenhagen, DK.

Os estudos selecionados foram os ensaios clínicos randomizados que fizeram comparação entre a drenagem e a não drenagem em cirurgia colorretal eletiva, em doentes adultos, sem restrição quanto ao tipo e ao material do dreno empregado. Assim, não foram considerados dados relativos ao local de drenagem, tipo de dreno utilizado e tempo de permanência do dreno.

A estratégia de busca dos estudos incluiu pesquisa nos bancos de dados computadorizados: EMBASE, LILACS, MEDLINE e registros de ensaios clínicos randomizados do Grupo de Câncer Colorretal da Cochrane Collaboration. O contato com os autores dos estudos selecionados foi realizado por meio de cartas, solicitando informações sobre estudos não publicados e relatos adicionais.

Quanto à seleção dos estudos, dois revisores, independentemente, avaliaram e selecionaram os ensaios clínicos incluídos na revisão, sem mascaramento dos revisores.

As discordâncias eram discutidas em reuniões de consenso, e se houvesse dúvida quanto à relevância de determinado ensaio clínico, o autor principal do estudo era contactado através de cartas e de acordo com sua resposta decidíamos sobre a inclusão ou não do estudo na revisão.

Quanto aos critérios de inclusão, os principais foram representados pelo ensaio clínico randomizado, que apresentasse sigilo de alocação mantido até o momento da intervenção.

$\mathrm{Na}$ avaliação crítica dos estudos outros critérios foram levados em consideração: princípio de intenção de tratar, descrição das perdas ou exclusões de participantes do estudo, 0 tempo de seguimento das variáveis analisadas pelos autores, descrição do cálculo do 
tamanho da amostra e a caracterização da representatividade da amostra. Quanto aos participantes, foram avaliados os critérios de inclusão e exclusão dos estudos, o tipo de doença, idade, sexo, local e tempo decorrido para a realização do estudo. Foram relacionadas as variáveis analisadas pelos autores com as respectivas unidades de medida.

Utilizou-se um programa de informática da Cochrane Collaboration (Review Manager, versão 4.04 para Windows, Oxford (UK): update software 1999), onde os dados relevantes foram coletados.

As variáveis consideradas relevantes para esta pesquisa, assim como as conceituações adotadas para as suas identificações, foram as seguintes:

- Mortalidade geral: número de mortes intra-hospitalares devido a causas gerais.

- Mortalidade específica: número de mortes intra-hospitalares devido a complicações anastomóticas.

- Deiscência anastomótica clínica: descarga de conteúdo intestinal pelo sítio da anastomose, com exteriorização através da ferida operatória ou de orifício de drenagem na parede abdominal ou existência de um abscesso em sua proximidade.

- Deiscência anastomótica radiológica: extravasamento de contraste na região peri-anastomótica no controle radiológico pós-operatório da anastomose.

- Deiscência anastomótica global: somatória das taxas de deiscência clínica e radiológica.

- Reoperação: reintervenção cirúrgica por complicação anastomótica.

- Infecção da ferida operatória: presença de secreção contaminada na ferida abdominal.

- Complicação infecciosa extra-abdominal: complicação infecciosa pós-operatória em localização extra-abdominal.

As variáveis mortalidade global, mortalidade específica, deiscência anastomótica clínica, deiscência anastomótica radiológica, deiscência anastomótica global, reoperação, infecção da ferida operatória e complicações infecciosas extra-abdominais foram analisadas como variáveis dicotômicas.

Quando apropriados, os estudos foram subagrupados para a realização da metanálise, de acordo com o nível anastomótico (intra ou extraperitoneal) e com o tipo de drenagem (ativa ou passiva).

\begin{tabular}{|c|c|c|c|c|}
\hline \multicolumn{5}{|c|}{ Quadro I - Relaçäo de estudos incluídos na revisão sistemática } \\
\hline Autores & $n$ & D+ & D- & Nível da Anastomose \\
\hline Hoffmanetal., 1987 & 60 & 28 & 32 & Intraperitoneal \\
\hline Menningenetal., 1989 & 99 & 51 & 48 & Intraperitoneal \\
\hline Hagmüller etal., 1990 & 113 & 60 & 53 & Intraperitoneal \\
\hline Merad etal., 1998 & 317 & 156 & $|6|$ & Intraperitoneal \\
\hline \multirow[t]{2}{*}{ Merad etal., 1999} & 492 & 184 & 176 & Intraperitoneal \\
\hline & & 63 & 69 & Extraperitoneal \\
\hline
\end{tabular}

n: tamanho da amostra D+:drenagem realizada D-: drenagem não realizada

A metanálise das variáveis dicotômicas foi realizada pelo método da diferença de risco no modelo randômico e a heterogeneidade estatística dos resultados dos estudos foi avaliada pelo cálculo do teste do Qui-quadrado com grau $\mathrm{N}$ de liberdade, quando $\mathrm{N}$ é igual ao número de estudos menos I.

A data da última busca dos ensaios clínicos foi dezembro de 200I, devendo esta revisão ser atualizada em bases anuais.

\section{Resultados}

Cinco ensaios clínicos foram incluídos na revisão sistemática e metanálise. (Quadrol)

Três estudos foram excluídos da revisão: Johnson et al. (1989) ${ }^{6}$, que apresentaram quebra do sigilo de alocação inadequada, escolha da data de nascimento dos pacientes para a alocação nos grupos de estudo e a não apresentação dos resultados de modo diferenciado para as anastomoses intra e extraperitoneal; Sagar et al. (1993) incluíram pacientes submetidos a cirurgias de emergência e Sagar et al. (1995) ${ }^{10}$, que não apresentaram resultados diferenciados para anastomoses intra e extraperitoneal, além de incluírem pacientes submetidos à cirurgia de emergência.

A mortalidade geral foi analisada em quatro estudos ${ }^{5,7,8,11}$, incluindo-se $589 \mathrm{pa}$ cientes, 295 no grupo de drenagem e 294 no grupo de não drenagem. A taxa de mortalidade geral foi de $3 \%$ ( $(9 / 295)$ e $5 \%$ (I5/294), respectivamente, não se observando diferença estatisticamente significante entre os grupos $(p=0,3078)$, com diferença de risco no modelo randômico de - $1 \%$, intervalo de confiança de $95 \%$, $4 \%$ a $1 \%$.
A mortalidade específica foi analisada em quatro estudos ${ }^{5,7,8,11}$, incluindo-se 589 pacientes, verificando-se taxas de mortalidade específica de $3 \%(8 / 295)$ no grupo com drenagem 2\% (7/294) no grupo sem drenagem, sem diferença estatisticamente significante entre os grupos $(p=0,704)$, com diferença de risco no modelo randômico de $0 \%$, intervalo de confiança de $95 \%$, de $-2 \%$ a $3 \%$.

A deiscência anastomótica clínica foi estudada em quatro estudos ${ }^{5,7,8,11}$, incluindose 587 pacientes, observando-se incidência de $1 \%(4 / 295)$ no grupo com drenagem e de $1 \%$ (4/292) no grupo sem drenagem, sem diferença estatisticamente significante entre os grupos $(p=0,8493)$, com diferença de risco no modelo randômico de $0 \%$, intervalo de confiança de $95 \%$, de $-2 \%$ a $2 \%$.

A deiscência anastomótica radiológica foi analisada em três estudo ${ }^{5,8,11}$, incluindose 470 doentes, verificando-se incidência de $2 \%$ (4/237) no grupo com drenagem e de $2 \%$ (4/233) no grupo sem drenagem, sem diferença estatisticamente significante entre os grupos $(p=0,6031)$, diferença de risco no modelo randômico de $0 \%$, intervalo de confiança de $95 \%$, de $-2 \%$ a $1 \%$.

A deiscência anastomótica global, somatória da deiscência clínica e da radiológica, foi estudada em 980 doentes, observando-se incidência de $5 \%$ (24/49I) no grupo com drenagem e de $4 \%$ (22/489) no grupo sem drenagem, sem diferença estatisticamente significante entre os grupos $(p=0,7949)$, diferença de risco no modelo randômico de $0 \%$, intervalo de confiança de $95 \%$, de $-2 \%$ a $2 \%$.

A reoperação, determinada com complicações anastomóticas, foi analisada em quatro estudos ${ }^{5,7,8,11}$, incluindo-se 589 pacientes, observando-se incidência de 4\% (I I/295) no 
Tabela I - Resultado da metanálise da variável deiscência anastomótica clínica por meio da diferença de risco com intervalo de confiança de $95 \%$

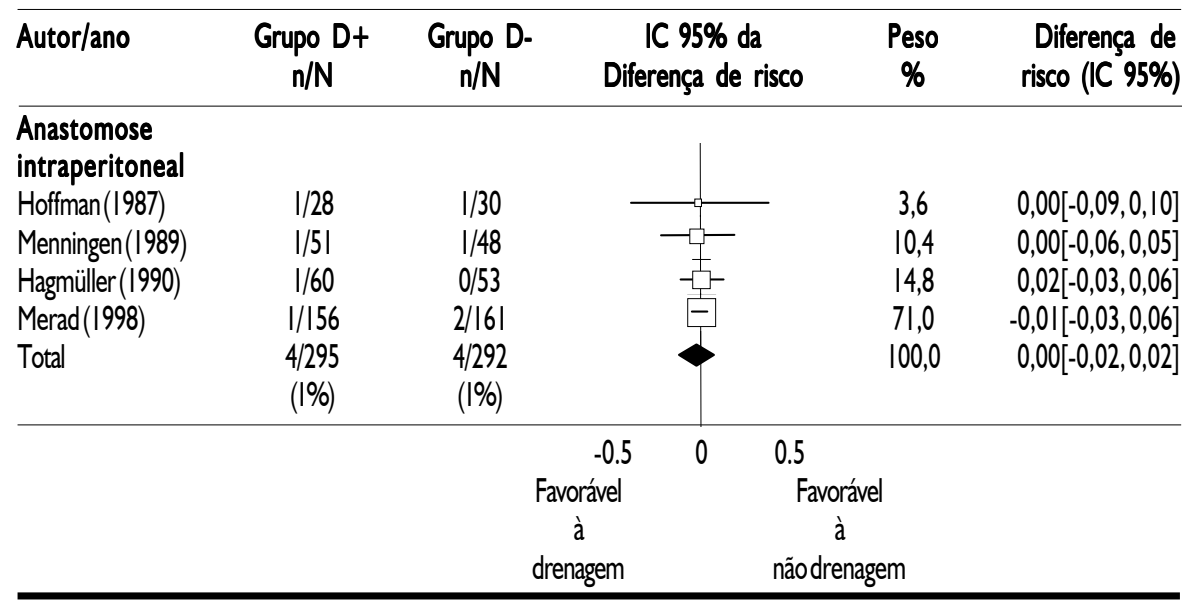

Teste de heterogeneidade dos dados: Qui-Quadrado 0.82; $\mathrm{df}=3 ; \mathrm{p}=0.87$.

D+:drenagem D-:nãodrenagemm IC:intervalodeconfiança n:númerodeeventos N:tamanhodogrupo DR: Diferença de risco, $p=0.5$.

\section{Quadro 2 - Resultados gerais das metanálises das variáveis analisadas}

0I Mortalidade geral (4 estudos 589 pacientes)

02Mortalidadeespecífica (4 estudos, 589 pacientes)

03 Deiscência clínica (4 estudos, 587 pacientes)

04Deiscênciaradiológica ( 3 estudos, 470 pacientes)

05 Deiscênciaglobal (4 estudos, 980 pacientes)

06 Reoperação (4 estudos, 580 pacientes)

07 Infecção ferida operatória (4 estudos, 587 pacientes)

08 Infecção extra-abdominal (estudos, 488 pacientes)

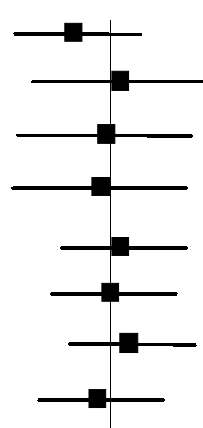

$-0,02[-0,05,0,01]$

$0,00[-0,02,0,03]$

$0.00[-0,02,0.02]$

$0,00[-0.03,0,02]$

$0,00[-0,02,0,03]$

$0,00[-0,03,0,03]$

$0,01[-0,02,0,04]$

$-0,01[-0,05,0,03]$

Quadro 3 - Distribuiçäo dos autores e amostras conforme o tipo (ativa ou passiva) e a via de drenagem

\begin{tabular}{lcc}
\hline $\begin{array}{l}\text { Autor e } \\
\text { amostra }\end{array}$ & $\begin{array}{c}\text { Tipo de } \\
\text { drenagem } \\
\text { Hoffmann, } 1987\end{array}$ & $\begin{array}{c}\text { Via de } \\
\text { drenagem }\end{array}$ \\
$\mathrm{N}=60$ & Passiva & Contra-abertura \\
$\begin{array}{l}\text { Menningen, } 1989 \\
\mathrm{~N}=99\end{array}$ & Passiva & Contra-abertura \\
Hagmuller, 1998 & Passiva & \\
$\mathrm{N}=113$ & Contra-abertura \\
Merad, 1998 & Ativa/Passiva \\
$\mathrm{N}=317$ & & Contra-abertura \\
Merad, 1999 & Ativa & \\
$\mathrm{N}=492$ & & Contra-abertura \\
\hline
\end{tabular}

grupo com drenagem e de 4\% (II/294) no grupo sem drenagem, não se observando diferença estatisticamente significante entre os grupos $(p=0,7339)$, diferença de risco no modelo randômico de $0 \%$, intervalo de confiança de $95 \%$, de $-2 \%$ a $3 \%$.

A infecção da ferida operatória foi analisada em quatro estudos ${ }^{5,7,8,11}$, incluindose 587 doentes, verificando-se incidência de $5 \%$ (I 4/295) no grupo com drenagem e de $4 \%$ (I I/292) no grupo sem drenagem, não se observando diferença estatisticamente significante entre os grupos $(p=0,8259)$, diferença de risco no modelo randômico de $1 \%$, intervalo de confiança de $95 \%$, de $-2 \%$ a $4 \%$.

A infecção extra-abdominal foi analisada em três estudos ${ }^{5,8,11}$, incluindo-se 488 doentes, tendo sido verificado incidência de $7 \%$ (I $7 / 244)$ no grupo com drenagem e de $8 \%$ (20/244) no grupo sem drenagem, sem diferença estatisticamente significante entre os grupos $(p=0,5353)$, diferença de risco no modelo randômico de $1 \%$, intervalo de confiança de $95 \%$, de $-5 \%$ a $3 \%$.

0 resultado da metanálise da deiscência anastomótica clínica, considerada como variável mais importante, e 0 respectivo gráfico podem ser analisados na Tabela I.

Os resultados das metanálises de todas as variáveis analisadas na revisão são apresentados no Quadro 2.

A análise da amostra conforme o tipo de drenagem, a via de drenagem (local de exteriorização na parede abdominal) podem ser observados no Quadro 3.

O resultado da metanálise da deiscência anastomótica clínica conforme o tipo de drenagem e o respectivo gráfico podem ser analisados na Tabela 2.

Os resultados das metanálises das variáveis analisadas, estratificando-se a amostra conforme o tipo de drenagem, são apresentados no Quadro 4.

Não estavam disponíveis nos trabalhos selecionados dados referentes ao tipo do dreno utilizado, ao local de posicionamento do dreno e ao tempo de permanência do dreno no local de drenagem. 
Tabela 2 - Resultado da metanálise da variável deiscência anastomótica clínica conforme o tipo de drenagem (ativa ou passiva), por meio da diferenç̧a de risco com intervalo de confiança de $95 \%$

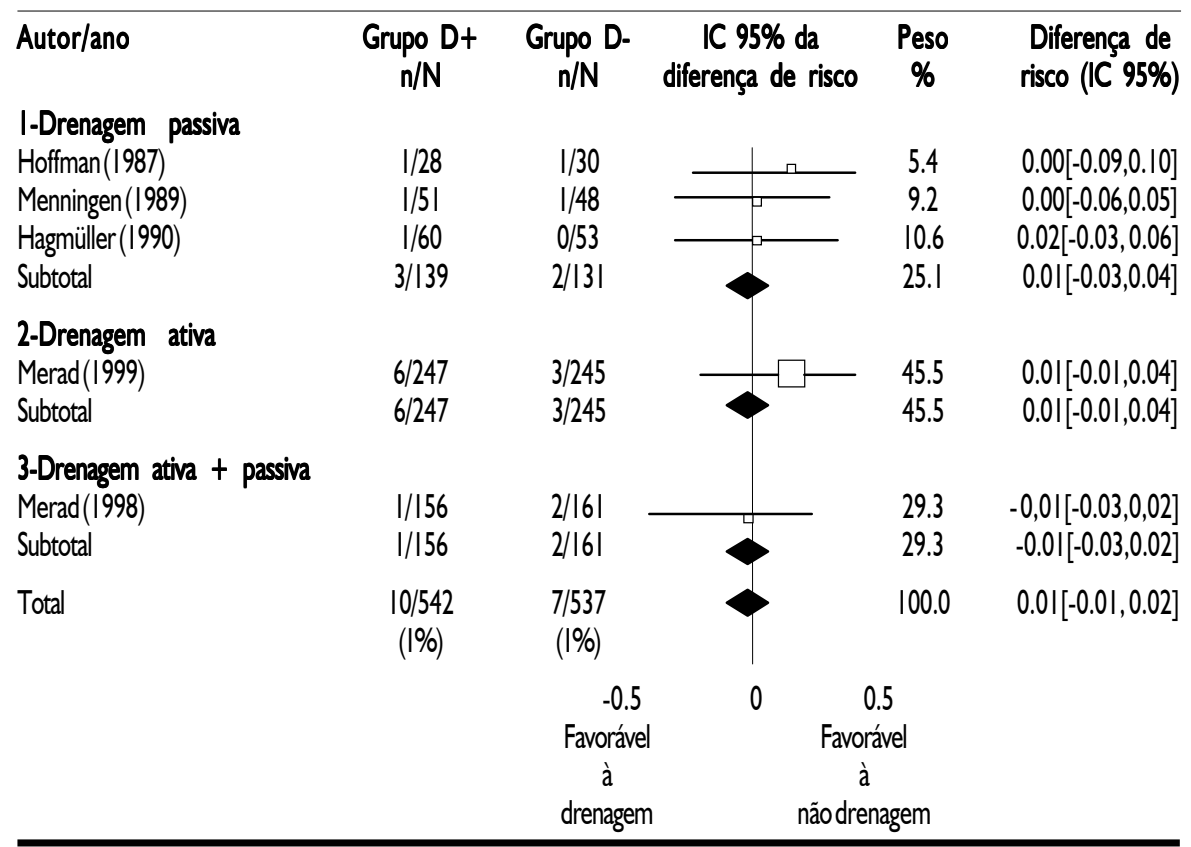

Teste de heterogeneidade dos dados: Qui-Quadrado I.00; $\mathrm{df}=4 ; \mathrm{p}=0,91$ )

D+:drenagem D-nãodrenagem IC:intervalodeconfiança n:númerode eventos N:tamanhodogrupo

DR: Diferença de risco, $p=0.3$.

Quadro 4 - Resultados gerais das metanálises das variáveis analisadas, com amostra estratificada conforme 0 tipo de drenagem

\begin{tabular}{|c|c|c|}
\hline Variáveis & Diferença de risco & (IC 95\%) \\
\hline OI Mortalidade geral(5 estudos, 108 I pacientes) & & $-0.01[-0.04,0.01]$ \\
\hline 02Mortalidade específica(5 estudos, 108 I pacientes) & & $0.00[-0.01,0.02]$ \\
\hline 03 Deiscênciaclínica(5 estudos, 1079 pacientes) & & $0.01[-0.01,0.02]$ \\
\hline 04 Deiscênciaradiológica (4 estudos, 962 pacientes) & & $0.00[-0.03,0.02]$ \\
\hline 05 Deiscênciaglobal (4 estudos, 980 pacientes) & $\rightarrow$ & $0.00[-0.02,0.03]$ \\
\hline 06Reoperação(5 estudos, 108 I pacientes) & $\longrightarrow$ & $0.01[-0.02,0.04]$ \\
\hline 07Infecçãoferida operatória(5estudos, 1079 pacientes) & & $0.00[-0.03,0.02]$ \\
\hline 08 Infecção extra-abdominal (4estudos,980 pacientes) & - & $0.00[-0.03,0.03]$ \\
\hline
\end{tabular}

\section{Discussão}

A drenagem profilática representa uma tentativa de prevenção, a partir do posicionamento de um dreno, da formação de coleções intra-abdominais. Tais coleções, conforme demonstrado por alguns autores ${ }^{17,18,19}$, podem representar fatores de complicações anastomóticas, como deiscência e estenose.

Adrenagem abdominal profilática de rotina representa conduta que não depende de fatores circunstanciais, como hemostasia não adequada, grau de contaminação da cavidade peritoneal, anastomoses de risco etc., que representam condições onde a drenagem é necessária.

$O$ questionamento sobre a drenagem profilática de rotina de anastomoses colorretais extraperitoneais torna-se mais relevante, dado o maior potencial destas anastomoses para a deiscência. Infeliz- mente, nesta revisão não encontramos resposta para essa dúvida, visto que a maior parte da amostra é constituída de anastomoses intraperitoneais. Apenas um autor $^{12}$ estratificou sua análise, estudando a variável deiscência anastomótica para anastomoses colorretais extraperitoneais, em 132 doentes, não tendo observado diferença significante entre o grupo drenado ${ }^{63} \mathrm{e} 0$ não-drenado ${ }^{69}$.

Pelo exposto, fica evidente a necessidade de se comprovar a eficácia da drenagem profilática de rotina de anastomoses colorretais extraperitoneais. Configurase, desta forma, uma outra pesquisa também de alta relevância.

Nesta revisão a variável mortalidade foi analisada em quatro $0^{5,78,11}$ dos cinco estudos selecionados, enfocando-se mortalidade intra-hospitalar geral e específica; esta última representa aquela decorrente de complicação da anastomose propriamente dita podendo, teoricamente, estar associada à utilização ou não de drenos abdominais. Nesta revisão, a drenagem não pareceu evitar a mortalidade específica, já que não houve diferenças entre os grupos estudados.

A variável deiscência anastomótica clínica foi analisada em quatro estudos $\mathrm{s}^{5,7,8,11} \mathrm{e}$ a metanálise realizada não demonstrou diferença significante entre os grupos drenados e não drenados, permitindo inferir que o procedimento de drenagem não impediu a ocorrência de uma deiscência anastomótica. $\mathrm{O}$ alegado fator de proteção, representado pelo dreno junto à anastomose ${ }^{20,21}$, não foi eficaz no sentido de diferenciar os resultados nos grupos analisados, quando se considerou a variável deiscência anastomótica clínica.

A deiscência anastomótica radiológica, analisada em três dos cinco estudos selecionados, também não demonstrou diferença significante entre os grupos drenado e não drenado, não sendo o dreno eficaz na prevenção da deiscência anastomótica radiológica.

A variável reoperação foi analisada em quatro $5,7,8,11$ dos cinco estudos selecionados, enfocou somente indicações de reoperação por complicação 
anastomótica; da mesma forma que as variáveis anteriores, ela também não demonstrou diferença significante entre os grupos drenados e não drenados. Infere-se que o dreno não exerceu o papel que, teoricamente, é imputado a ele, ou seja, permitir o escape do conteúdo intestinal pelo orifício da parede abdominal, impedindo, assim, a disseminação para a cavidade peritoneal, quando da deiscência anastomótica' ${ }^{19}$.

Com base nestes resultados, a afirmação de que os drenos abdominais evitam as reoperações, quando de complicações anastomóticas, carece de sustentação científica.

Os resultados desta revisão sistemática e metanálise mostraram que a infecção da ferida operatória, analisada em quatro ${ }^{5,7,8,11}$ de cinco estudos selecionados, não está associada à presença ou ausência do dreno abdominal.

Teoricamente, o dreno deveria permitir o escoamento dos fluídos da cavidade peritoneal, poupando a cicatriz operatória e, portanto, minimizando a incidência de infecção local, fato que não foi observado.

A partir dos resultados desta revisão e metanálise, pode-se inferir que, do ponto de vista da infecção da ferida operatória, a drenagem rotineira e profilática de anastomoses intraperitoneais, na cirurgia colorretal, pode ser questionada.

A variável complicações infecciosas extra-abdominais foi estudada em três ${ }^{5,8,11}$ dos cinco estudos selecionados, não tendo também apresentado diferença significante entre os grupos drenado e não drenado. Assim, a presença do dreno não alterou a incidência de complicações como infecção pulmonar, infecção do trato urinário etc. no pós-operatório.

Como não foram detectadas vantagens na utilização da drenagem ou não, profilática de rotina, em anastomoses intraperitoneais na cirurgia colorretal eletiva, esta questão permanece em aberto. A utilização de drenos profiláticos de rotina não é, portanto, justificável. Ressalva-se, no entanto, que do ponto de vista prático, quando da identificação de um ou mais fatores de risco de deiscência anastomótica, a decisão deve ser baseada na experiência pessoal e no julgamento adequado das condições intra-operatórias para a adoção da melhor conduta.
Quando procedeu-se a análise dos resultados conforme o tipo de drenagem empregado, se passiva ou ativa, também não foram observadas diferenças significantes nas variáveis. Aestratificação conforme o tipo de drenagem permitiu maior homogenização da amostra implicando, no entanto, redução do tamanho amostral, com perda do poder estatístico. A maioria dos autores utilizou drenos de látex, posicionados por contra-abertura. Estes dados, no entanto, não foram uniformes, visto que o tipo de dreno, o local de drenagem e o tempo de permanência do dreno não foram relatados adequadamente.

No que diz respeito à pesquisa, a primeira implicação resultante desta revisão sistemática e metanálise é que novas pesquisas devem ser realizadas para as anastomoses colorretais extra-peritoneais, no sentido de se aumentar o número de participantes, o que permitiria detectar diferenças significantes, caso estas existam.

Sugerimos, com o intuito de se obter respostas confiáveis e que possam contribuir para a tomada de decisão nesta área médica, a realização de ensaios clínicos com objetivo bem definido e dirigido especificamente para anastomoses com o mesmo potencial de risco de deiscência, com processo de randomização adequado, explicitamente descrito no trabalho e com o momento da quebra do sigilo de alocação bem definido, descrevendo também as perdas e exclusões de participantes do estudo. No sentido de se homogeneizar os grupos a serem comparados, a seleção adequada dos participantes torna-se necessária, não devendo portanto, incluir pacientes atendidos em condições emergenciais. Da mesma forma, o tipo de dreno, o local de posicionamento do dreno e o seu tempo de permanência devem ser levados em consideração.

\section{ConClusÃo}

Não se considerando o tipo, o local de posicionamento e o tempo de permanência do dreno, os resultados da presenterevisão sistemática e metanálise permitem concluir que as evidências encontradas são insuficientes para demonstrar diferenças significantes entre drenagem ou não, profilática de rotina, deanastomoses intraperitoneais em cirurgia colorretal eletiva.

\begin{abstract}
SUMmaRY
ProphyLACTIC ROUTINE ANASTOMOTIC DRAINAGE IN ELECTIVE COLORECTAL SURGERY. SYSTEMATIC REVIEW AND METANALYSIS

OBJECTIVE. To analyse the results of the comparison of the use or not of routine prophylactic drainage of anastomoses after elective colorectal surgery. The following hypothesis will be tested: the use of routine prophylactic drainage of anastomoses after elective colorectal surgery is unjustified. [Rev Assoc Med Bras 2003; 49(2): 214-9]
\end{abstract}

KEY WORDS: Surgical drainage. Colorectal surgery. Systematic review. Metaanalysis. Surgical anastomosis.

\section{REFERÊNCIAS}

I. Berliner SD, Burson LC, Lear PE. Use and abuse of intraperitoneal drains in colon surgery. Arch. Surg. 1964; 89:686-90.

2. Canalis F, Ravitch MM. Study of healing of inverting and everting intestinal anastomoses. Surg Gynecol Obstet 1968; 126:109-14.

3. Manz CW, La Tendresse C, Sako Y. The detrimental effects of drains on colonic anastomosis: na experimental study. Dis Colon Rectum 1970; 13:17-25.

4. Crowson WN, Wilson CS. An experimental study of the effects of drains on colon anastomoses. Am. Surg. 1973; 39: 597-601.

5. Hoffmann J, Shokouh-Amiri MH, Damm P, Jensen R. A prospective controlled study of prophylactic drainage after colonic anastomoses. Dis Colon Rectum 1 987; 30:449-52.

6. Johnson CD, Lamont PM, Orr N, Lennox M. Is a drain necessary after colonic anastomosis ?J R Soc Med 1989; 82:66I-4.

7. Menningen R, Kusche J, Troidl H. Prophylaktische drainage von Kolonanastomosen. Coloproctology 1989; I I:76-80.

8. Hagmüller E, Lorenz D, Werthmann K, Trede M. Uses and risks of drainage following elective colon ressection. A prospective, randomized and controlled clinical study. Chirurg 1990; 61:266-71.

9. Sagar PM, Couse N, Kerin N, May J, Mc Fie J. Randomized trial of drainage of colorectal anastomosis. Br J Surg 1 993; 80:769-7I.

10. Sagar PM, Hartley MN, Mc Fie J, ManleyJones B, Sedman P, May J. Randomized trial of pelvic drainage after rectal resection. Dis Colon Rectum 1 995; 38:254-8.

I I. Merad F, Yahchouchi E, Hay JM, Fingerhut A, Laborde Y, Langlois-Zantain $O$. Prophylactic abdominal drainage after elective colonic resection and suprapromontory anastomosis: a multicenter study controlled by randomization. Arch Surg 1998; 133:309- I 4. 
12. Merad F, Hay JM, Fingerhut A, Yahchouchi $E$, Laborde $Y$, Pélissier E, et al. Is prophylactic pelvic drainage useful after elective rectal or anal anastomosis? A multicenter controlled randomized trial. Surgery 1999; 125:529-35.

13. Peto R. Why do we need systematic overviews of randomized trials ? Stat Med 1987; 6:233-40.

14. Collins R, Peto R, Gray R, Parish S. Largescale randomized evidence: trials and overviews. In: Weatherall DJ, Ledingham JGG, Warrell DA. Oxford textbook of medicine. 3rd ed. Oxford: Oxford University Press; 1996. p.21-32.

15. Guyatt GH, Cook DJ, Sackett DL, Eckman
MH, Pauker SG. Grades of recomendation for antithrombotic agents. Chest Supplement 1998; I I4:44 I-4.

16. Mulrow CD, Oxman AD. Cochrane Collaboration Handbook. Oxford: Cochrane Library.; 1997.

17. Beahrs $\mathrm{OH}$. Low anterior resection for cancer of the rectosigmoid and rectum. Surg Clin North Am 1967; 47:97I -5.

18. Gallagher P, Cade P, Whale R, Scholefield $P F$. Does infection cause anastomotic dehiscence? J R Coll Surg Edinb 1982; 27:90-2.

19. Gingold BS, Jagelman DG. Value of pelvic suction- irrigation in reducing morbity of low anterior resection of the rectum. A ten- year experience. Surgery 1982; 97:394-98.

20. Collins $\mathrm{CD}$, Talbot $\mathrm{CH}$. Pelvic drainage after anterior resection of the rectum. Arch Surg 1969; 99:391-3

21. Averback AM, Sugarbaker PH. The use of drains in elective surgery for colorectal cancer: always, never or selectively ? Tumori I 995; 8I (suppl.):87-9.

Artigo recebido: 16/04/2002 Aceito para publicação: 22/10/2002 